\title{
Existence of positive solutions for a fractional high-order three-point boundary value problem
}

\author{
Guoqing Chai* and Songlin $\mathrm{Hu}$
}

\section{"Correspondence:}

mathchgq@163.com

College of Mathematics and

Statistics, Hubei Normal University,

Hubei, 435002, P.R. China

\begin{abstract}
In this paper, the authors consider the following fractional high-order three-point boundary value problem: $D_{0^{+}}^{\alpha} u(t)+f(t, u(t))=0, t \in(0,1), u(0)=u^{\prime}(0)=\cdots=$ $u^{(n-2)}(0)=0, D_{0^{+}}^{\alpha-1} u(\eta)=k D_{0^{+}}^{\alpha-1} u(1)$, where $k>1, \eta \in(0,1), n-1<\alpha \leq n, n \geq 3, D_{0^{+}}^{\alpha}$ is the standard Riemann-Liouville derivative of order $\alpha$, and

$f:[0,1] \times[0,+\infty) \rightarrow[0,+\infty)$ is continuous. By using some fixed point index theorems on a cone for differentiable operators, the authors obtain the existence of positive solutions to the above boundary value problem.
\end{abstract}

MSC: 34A08; 34B15

Keywords: fractional differential equations; three-point boundary value problems; existence results; fixed point index theorem for differentiable operators

\section{Introduction}

In this paper, we investigate the existence of solutions for the following fractional highorder equation:

$$
D_{0^{+}}^{\alpha} u(t)+f(t, u(t))=0, \quad t \in(0,1)
$$

with the three-point boundary value conditions

$$
u(0)=u^{\prime}(0)=\cdots=u^{(n-2)}(0)=0, \quad D_{0^{+}}^{\alpha-1} u(\eta)=k D_{0^{+}}^{\alpha-1} u(1),
$$

where $k>1, \eta \in(0,1), n-1<\alpha \leq n, n \geq 3, D_{0^{+}}^{\alpha}$ is the standard Riemann-Liouville derivative of order $\alpha$ and $f:[0,1] \times[0,+\infty) \rightarrow[0,+\infty)$ is continuous.

Differential equations with fractional order are a generalization of the ordinary differential equations to non-integer order. This generalization is not a mere mathematical curiosity but rather has interesting applications in many areas of science and engineering such as electrochemistry, control, porous media, electromagnetism, etc. (see [1-5]). There has been a significant development in the study of fractional differential equations in recent years; see for example [6-27]. Furthermore, several kinds of the high-order boundary value problems of fractional equations have been studied; see [6-10, 28-31] for example. In [28], using the Guo-Krasnosel'skii fixed point theorem, Goodrich discussed the exis- 
tence of positive solutions for the following fractional boundary value problem:

$$
\left\{\begin{array}{l}
D_{0^{+}}^{v} u(t)+f(t, u(t))=0, \quad 0<t<1, n-1 \leq v \leq n, \\
u^{(i)}(0)=0, \quad 0 \leq i \leq n-2, \\
\left.D_{0^{+}}^{\alpha} u(t)\right|_{t=1}=0, \quad 1 \leq \alpha \leq n-2,
\end{array}\right.
$$

where $f \in C([0,1] \times[0,+\infty),[0,+\infty)), n>3$.

Moreover, Goodrich [29] investigated the existence of a positive solution to system of fractional boundary value problems and extended his previous study in [28].

Recently, motivated by the above work of Goodrich, Xu et al. [6] investigated the existence and uniqueness of positive solution for the following fractional boundary value problem:

$$
\left\{\begin{array}{l}
D_{0^{+}}^{v} u(t)+h(t) f(t, u(t))=0, \quad 0<t<1, n-1<v \leq n \\
u^{(i)}(0)=0, \quad 0 \leq i \leq n-2, \\
\left.D_{0^{+}}^{\alpha} u(t)\right|_{t=1}=0, \quad 1 \leq \alpha \leq n-2
\end{array}\right.
$$

where $f \in C([0,1] \times[0,+\infty),[0,+\infty)), h \in C(0,1) \cap L(0,1)$ and $n>3$.

More recently, by the method of upper and lower solution together with Schauder fixed position theorem, Vong [7] studied the existence of positive solutions of the nonlocal boundary value problem for fractional equation:

$$
\left\{\begin{array}{l}
D_{0^{+}}^{\alpha} u(t)+f(t, u(t))=0, \\
u^{\prime}(0)=\cdots=u^{(n-1)}(0)=0, \quad u(1)=\int_{0}^{1} u(s) d \mu(s),
\end{array}\right.
$$

where $n \geq 2, \alpha \in(n-1, n)$, and $\mu(s)$ is a function of bounded variation.

Moreover, Waug et al. [30], El-Shahed and Shammakh [31], Yang et al. [8], Zhang and Han [9], Wu et al. [10] also studied similar problems.

It is worth pointing out that the fixed point index theorems on cone for differentiable operators are the effective tools to investigate positive solutions of fractional equation. However, to the author's knowledge, such theorems are rarely used in the literature. Different from the literature mentioned above, in the present paper, the authors apply some fixed point theorems for differentiable operators to establish the existence results on positive solutions to the fractional nonlocal boundary value problem (1.1)-(1.2). That is one of the features of this paper. Another feature of this paper is that some spectral properties of a correlative linear integral operator are introduced to obtain some positive eigenvector.

The rest of this paper is organized as follows. In Section 2, we present some necessary definitions and preliminary results that will be used to prove our main results. In Section 3, we put forward and prove our main results. Finally, we will give two examples to demonstrate our main results.

\section{Preliminaries}

In this section, we introduce some preliminary facts which are used throughout this paper.

Let $\mathbb{N}$ be the set of positive integers, $\mathbb{R}$ be the set of real numbers. $\mathbb{R}_{+}$be the set of real positive numbers. Denote by $C([a, b], \mathbb{R})$ the Banach space endowed with the norm $\|u\|=\max _{t \in[a, b]}|u(t)|$. Let $E=C([0,1], \mathbb{R})$. 
Definition 2.1 ([3]) The Riemann-Liouville fractional integral of order $\alpha>0$ of a function $y:(a, b] \rightarrow \mathbb{R}$ is given by

$$
I_{a^{+}}^{\alpha} y(t)=\frac{1}{\Gamma(\alpha)} \int_{a}^{t}(t-s)^{\alpha-1} y(s) d s, \quad t \in(a, b] .
$$

Definition 2.2 ([3]) The Riemann-Liouville fractional derivative of order $\alpha>0$ of function $y:(a, b] \rightarrow \mathbb{R}$ is given by

$$
D_{a^{+}}^{\alpha} y(t)=\frac{1}{\Gamma(n-\alpha)}\left(\frac{d}{d t}\right)^{n} \int_{a}^{t} \frac{y(s) d s}{(t-s)^{\alpha-n+1}}, \quad t \in(a, b],
$$

where $n=[\alpha]+1,[\alpha]$ denotes the integer part of $\alpha$.

Lemma 2.1 ([32]) Let $\alpha>0$. If $u \in C(0,1) \cap L(0,1)$ with a fractional derivative of order $\alpha$ that belongs to $C(0,1) \cap L(0,1)$, then

$$
I_{0^{+}}^{\alpha} D_{0^{+}}^{\alpha} u(t)=u(t)+c_{1} t^{\alpha-1}+c_{2} t^{\alpha-2}+\cdots+c_{n} t^{\alpha-n},
$$

for some $c_{i} \in \mathbb{R}, i=1,2, \ldots, n, n-1<\alpha \leq n$.

To study the existence of solutions to the boundary value problems (BVPs, for short), we first consider the following auxiliary BVP:

$$
\left\{\begin{array}{l}
D_{0^{+}}^{\alpha} u(t)+h(t)=0 \\
u(0)=u^{\prime}(0)=\cdots=u^{(n-2)}(0)=0, \quad D_{0^{+}}^{\alpha-1} u(\eta)=k D_{0^{+}}^{\alpha-1} u(1),
\end{array}\right.
$$

where $h \in E$, and $\alpha, \eta, k, n$ are given in (1.2).

We have the following lemma.

Lemma 2.2 For $h \in E$, the BVP (2.1) has a unique solution given by

$$
u(t)=\int_{0}^{1} G(t, s) h(s) d s, \quad t \in[0,1]
$$

where

$$
G(t, s)=\frac{1}{\Gamma(\alpha)}\left\{\begin{array}{l}
t^{\alpha-1}-(t-s)^{\alpha-1}, \quad 0 \leq s \leq \min \{\eta, t\}, \\
t^{\alpha-1}, \quad t \leq s \leq \eta, \quad \\
\frac{k}{k-1} t^{\alpha-1}-(t-s)^{\alpha-1}, \quad \eta<s \leq t \\
\frac{k}{k-1} t^{\alpha-1}, \quad \max \{\eta, t\}<s \leq 1 .
\end{array}\right.
$$

Proof By Lemma 2.1, it follows from $h \in E$ that there exist some constants $c_{k}(1 \leq k \leq n)$ such that

$$
u(t)=-I_{0^{+}}^{\alpha} h(t)+c_{1} t^{\alpha-1}+c_{2} t^{\alpha-2}+\cdots+c_{n} t^{\alpha-n}, \quad t \in[0,1] .
$$

The boundary value conditions $u(0)=u^{\prime}(0)=\cdots=u^{(n-2)}(0)=0$ in (2.1) imply that $c_{2}=$ $c_{3}=\cdots=c_{n}=0$, and so

$$
u(t)=-I_{0^{+}}^{\alpha} h(t)+c_{1} t^{\alpha-1}, \quad t \in[0,1] .
$$


Hence

$$
D_{0^{+}}^{\alpha-1} u(t)=-\int_{0}^{t} h(s) d s+c_{1} \Gamma(\alpha), \quad t \in[0,1]
$$

Thus,

$$
\begin{aligned}
& D_{0^{+}}^{\alpha-1} u(\eta)=-\int_{0}^{\eta} h(s) d s+c_{1} \Gamma(\alpha), \\
& D_{0^{+}}^{\alpha-1} u(1)=-\int_{0}^{1} h(s) d s+c_{1} \Gamma(\alpha) .
\end{aligned}
$$

The condition $D_{0^{+}}^{\alpha-1} u(\eta)=k D_{0^{+}}^{\alpha-1} u(1)$ together with (2.4) and (2.5) yields

$$
c_{1}=\frac{(k-1) \int_{0}^{\eta} h(s) d s+k \int_{\eta}^{1} h(s) d s}{(k-1) \Gamma(\alpha)}
$$

and so

$$
u(t)=-I_{0^{+}}^{\alpha} h(t)+\frac{(k-1) \int_{0}^{\eta} h(s) d s+k \int_{\eta}^{1} h(s) d s}{(k-1) \Gamma(\alpha)} t^{\alpha-1}, \quad t \in[0,1]
$$

from (2.3).

(1) If $0 \leq t \leq \eta$, then

$$
\begin{aligned}
u(t)= & \frac{1}{\Gamma(\alpha)}\left[\int_{0}^{t}\left(t^{\alpha-1}-(t-s)^{\alpha-1}\right) h(s) d s\right. \\
& \left.+\int_{t}^{\eta} t^{\alpha-1} h(s) d s+\frac{k}{k-1} \int_{\eta}^{1} t^{\alpha-1} h(s) d s\right] \doteq T_{1} h(t) .
\end{aligned}
$$

(2) If $t \geq \eta$, then

$$
\begin{aligned}
u(t)= & \frac{1}{\Gamma(\alpha)}\left[\int_{0}^{\eta}\left(t^{\alpha-1}-(t-s)^{\alpha-1}\right) h(s) d s\right. \\
& \left.+\int_{\eta}^{t}\left(\frac{k}{k-1} t^{\alpha-1}-(t-s)^{\alpha-1}\right) h(s) d s+\frac{k}{k-1} \int_{t}^{1} t^{\alpha-1} h(s) d s\right] \doteq T_{2} h(t) .
\end{aligned}
$$

So, we always have

$$
u(t)=\int_{0}^{1} G(t, s) h(s) d s, \quad t \in[0,1]
$$

where Green's function $G(t, s)$ is given by (2.2). The proof is complete.

Now, we give some properties of $G(t, s)$.

Lemma 2.3 The Green function $G$ has the following properties:

(1) $G(t, s)>0$ for all $t, s \in(0,1]$;

(2) $\min _{t \in[\eta, 1]} G(t, s) \geq \omega_{0} G(\tau, s)$ for all $s, \tau \in[0,1]$, where $\omega_{0}=\frac{\eta^{\alpha-1}}{1+k_{0}}$ and $k_{0}=\frac{1}{(k-1)\left(1-(1-\eta)^{\alpha-1}\right)}$. 
Proof It is easy to see that the conclusion (1) of Lemma 2.3 is true from the expression of $G(t, s)$ in (2.2). So, it remains to show that the conclusion (2) of Lemma 2.3 is true. Our proof is divided into two steps.

Step 1. In this step, we show that

$$
\min _{t \in[\eta, 1]} G(t, s) \geq \frac{1}{\Gamma(\alpha)} \eta^{\alpha-1}\left(1-(1-s)^{\alpha-1}\right), \quad s \in[0,1]
$$

Let $\phi(t, s)=\Gamma(\alpha) G(t, s), t \in[\eta, 1], s \in[0,1]$. Then we have the following cases to consider.

(i) If $s \in[0, \eta]$, then

$$
\begin{aligned}
\phi(t, s) & =t^{\alpha-1}-(t-s)^{\alpha-1}=t^{\alpha-1}\left[1-\left(1-\frac{s}{t}\right)^{\alpha-1}\right] \geq t^{\alpha-1}\left[1-(1-s)^{\alpha-1}\right] \\
& \geq \eta^{\alpha-1}\left[1-(1-s)^{\alpha-1}\right] .
\end{aligned}
$$

(ii) If $s \in(\eta, t]$, then

$$
\phi(t, s)=\frac{k}{k-1} t^{\alpha-1}-(t-s)^{\alpha-1}>t^{\alpha-1}-(t-s)^{\alpha-1} \geq t^{\alpha-1}\left[1-(1-s)^{\alpha-1}\right] \geq \eta^{\alpha-1}\left[1-(1-s)^{\alpha-1}\right],
$$

because $k>1$.

(iii) If $s \in(t, 1]$, then

$$
\phi(t, s)=\frac{k}{k-1} t^{\alpha-1}>\frac{k}{k-1} \eta^{\alpha-1}>\frac{k}{k-1} \eta^{\alpha-1}\left[1-(1-s)^{\alpha-1}\right]>\eta^{\alpha-1}\left[1-(1-s)^{\alpha-1}\right] .
$$

So, from the above analysis, we know that the inequality (2.8) holds.

Step 2. Now, we show that

$$
G(\tau, s) \leq \frac{1}{\Gamma(\alpha)}\left(1+k_{0}\right)\left[1-(1-s)^{\alpha-1}\right], \quad \tau, s \in[0,1]
$$

Let $\phi(\tau, s)=\Gamma(\alpha) G(\tau, s), \tau, s \in[0,1]$. Similar to the proof in Step 1, we deduce the relation (2.9).

(i) If $0 \leq s \leq \min \{\eta, \tau\}$, then $\phi(\tau, s)=\tau^{\alpha-1}-(\tau-s)^{\alpha-1}$. Owing to that $\alpha>2$, it is easy to know that

$$
\tau^{\alpha-1}-(\tau-s)^{\alpha-1} \leq 1-(1-s)^{\alpha-1}
$$

and so

$$
\phi(\tau, s) \leq 1-(1-s)^{\alpha-1} .
$$

(ii) If $\max \{\eta, \tau\}<s$, then

$$
\phi(\tau, s)=\frac{k}{k-1} \tau^{\alpha-1} \leq \frac{k}{k-1} s^{\alpha-1}<\frac{k}{k-1}\left[1-(1-s)^{\alpha-1}\right] \leq\left(1+k_{0}\right)\left[1-(1-s)^{\alpha-1}\right]
$$

noting that $s^{\alpha-1}+(1-s)^{\alpha-1} \leq 1$ for $s \in(0,1]$ and $k_{0}=\frac{1}{(k-1)\left(1-(1-\eta)^{\alpha-1}\right)}$. 
(iii) If $\tau \leq s \leq \eta$, then

$$
\phi(\tau, s)=\tau^{\alpha-1} \leq s^{\alpha-1} \leq 1-(1-s)^{\alpha-1}
$$

noting that $s^{\alpha-1}+(1-s)^{\alpha-1} \leq 1$ for $s \in[0,1)$.

(iv) If $\eta<s \leq \tau$, then

$$
\phi(\tau, s)=\frac{k}{k-1} \tau^{\alpha-1}-(\tau-s)^{\alpha-1} .
$$

From

$$
\phi_{\tau}^{\prime}(\tau, s)=(\alpha-1)\left[\frac{k}{k-1} \tau^{\alpha-2}-(\tau-s)^{\alpha-2}\right] \geq 0,
$$

we have

$$
\begin{aligned}
\phi(\tau, s) & \leq \phi(1, s)=\frac{k}{k-1}-(1-s)^{\alpha-1}=1-(1-s)^{\alpha-1}+\frac{1}{k-1} \\
& =1-(1-s)^{\alpha-1}+k_{0}\left(1-(1-\eta)^{\alpha-1}\right) \\
& <\left(1+k_{0}\right)\left[1-(1-s)^{\alpha-1}\right]
\end{aligned}
$$

noting that $k_{0}=\frac{1}{(k-1)\left(1-(1-\eta)^{\alpha-1}\right)}$.

Summing up the above discussion, we know that the inequality (2.9) holds.

Now, from (2.8) and (2.9), the conclusion (2) of Lemma 2.3 follows. The proof is complete.

We introduce a cone $P \subset E$ as follows:

$$
P=\left\{u \in E: u(t) \geq 0, t \in[0,1] ; u(t) \geq \omega_{0}\|u\| \text { for all } t \in[\eta, 1]\right\} .
$$

Define an operator $T: E \rightarrow E$ by

$$
T h=\int_{0}^{1} G(t, s) h(s) d s, \quad \text { for } h \in E .
$$

We establish the following lemma, which will be used in the next section.

Lemma 2.4 $T: P \rightarrow P$ is completely continuous.

Proof Let $B$ be an arbitrary bounded set in $E$. Then there exists $M>0$ such that $\|h\| \leq M$ for any $h \in B$. First, we show that the set $G=\{w \mid w=T h, h \in B\}$ is equicontinuous on $[0,1]$.

In fact, for an arbitrary $\varepsilon>0$ and any $h \in B$ as well as $t_{1}, t_{2} \in[0,1]$ with $t_{1}<t_{2}$, there are three cases to consider.

Case 1. If $t_{1}<t_{2} \leq \eta$, then from (2.6) and (2.10), it follows that

$$
\begin{aligned}
& \left|T h\left(t_{2}\right)-T h\left(t_{1}\right)\right| \\
& \quad=\left|T_{1} h\left(t_{2}\right)-T_{1} h\left(t_{1}\right)\right|
\end{aligned}
$$




$$
\begin{aligned}
\leq & \frac{1}{\Gamma(\alpha)}\left[\left|\int_{0}^{t_{2}}\left(t_{2}^{\alpha-1}-\left(t_{2}-s\right)^{\alpha-1}\right) h(s) d s-\int_{0}^{t_{1}}\left(t_{1}^{\alpha-1}-\left(t_{1}-s\right)^{\alpha-1}\right) h(s) d s\right|\right. \\
& \left.+\left|\int_{t_{2}}^{\eta} t_{2}^{\alpha-1} h(s) d s-\int_{t_{1}}^{\eta} t_{1}^{\alpha-1} h(s) d s\right|+\frac{k}{k-1}\left|\int_{\eta}^{1}\left(t_{2}^{\alpha-1}-t_{1}^{\alpha-1}\right) h(s) d s\right|\right] \\
\leq & \frac{M}{\Gamma(\alpha)}\left[\frac{3 k-2}{k-1}\left(t_{2}^{\alpha-1}-t_{1}^{\alpha-1}\right)+2\left(t_{2}-t_{1}\right)\right] .
\end{aligned}
$$

Therefore, there is a $\delta_{1}>0$ such that

$$
\left|T h\left(t_{2}\right)-\operatorname{Th}\left(t_{1}\right)\right|<\frac{\varepsilon}{2}, \quad \text { when } 0<t_{2}-t_{1}<\delta, t_{1}<t_{2} \leq \eta .
$$

Case 2. If $\eta \leq t_{1}<t_{2}$, then by a similar argument to (2.11), from (2.7) and (2.10), we have

$$
\begin{aligned}
\left|T h\left(t_{2}\right)-T h\left(t_{1}\right)\right| & =\left|T_{2} h\left(t_{2}\right)-T_{2} h\left(t_{1}\right)\right| \\
& \leq \frac{M}{\Gamma(\alpha)}\left[\frac{3 k-1}{k-1}\left(t_{2}^{\alpha-1}-t_{1}^{\alpha-1}\right)+\frac{2 k}{k-1}\left(t_{2}-t_{1}\right)\right] .
\end{aligned}
$$

Thus, there exists a $\delta_{2}>0$ such that

$$
\left|T h\left(t_{2}\right)-T h\left(t_{1}\right)\right|<\frac{\varepsilon}{2}, \quad \text { when } 0<t_{2}-t_{1}<\delta_{2}, \eta \leq t_{1}<t_{2} .
$$

Case 3. If $t_{1}<\eta<t_{2}$ with $0<t_{2}-t_{1}<\delta=\min \left\{\delta_{1}, \delta_{2}\right\}$, then from (2.12)-(2.13), it follows that

$$
\left|\operatorname{Th}\left(t_{2}\right)-\operatorname{Th}\left(t_{1}\right)\right| \leq\left|\operatorname{Th}\left(t_{2}\right)-\operatorname{Th}(\eta)\right|+\left|\operatorname{Th}(\eta)-T h\left(t_{1}\right)\right|<\varepsilon
$$

noting that $T_{1} h(\eta)=T_{2} h(\eta)$.

Summing up the above analysis on Cases 1-3, we conclude that $\left|T h\left(t_{2}\right)-T h\left(t_{1}\right)\right|<\varepsilon$ when $0<t_{2}-t_{1}<\delta=\min \left\{\delta_{1}, \delta_{2}\right\}$, for $t_{1}, t_{2} \in[0,1]$, that is, $G$ is equicontinuous on $[0,1]$.

Now, we show that $G$ is bounded in $E$.

In fact, from the fact that $0 \leq G(t, s) \leq \frac{k}{k-1}, t, s \in[0,1]$, we immediately have

$$
|T h(t)| \leq \int_{0}^{1} G(t, s)|h(s)| d s \leq \frac{k}{k-1} M, \quad t \in[0,1], \text { for all } h \in E .
$$

So, by the Arzela-Ascoli theorem, we know that $T: E \rightarrow E$ is a compact operator. Again, because $T$ is a bounded operator on $E$ owing to (2.14), $T: E \rightarrow E$ is continuous, and therefore $T$ is completely continuous on $E$.

Finally, we apply the Lemma 2.3 to obtain

$$
\operatorname{Th}(t)=\int_{0}^{1} G(t, s) h(s) d s \geq 0, \quad t \in[0,1]
$$

and

$$
\min _{t \in[\eta, 1]} \operatorname{Th}(t)=\min _{t \in[\eta, 1]} \int_{0}^{1} G(t, s) h(s) d s \geq \omega_{0} \int_{0}^{1} G(\tau, s) h(s) d s=\omega_{0} \operatorname{Th}(\tau), \quad \tau \in[0,1]
$$

for any $h \in P$. So, $\min _{t \in[\eta, 1]}(T h)(t) \geq\|T h\|$, that is, $T: P \rightarrow P$. The proof is complete. 
For the remainder of this section, we introduce the following lemmas, which will be used to obtain our main result in the next section.

Lemma 2.5 ([33]) Let $P$ be a cone in a Banach space $E, A: P \rightarrow$ P be completely continuous, and $A \theta=\theta$. Suppose that $A$ is differentiable at $\theta$ along $P$ and 1 is not an eigenvalue of $A_{+}^{\prime}(\theta)$ corresponding to a positive eigenvector. Moreover, if $A_{+}^{\prime}(\theta)$ has no positive eigenvectors corresponding to an eigenvalue greater than one, then there exists $r_{0}>0$ such that

$$
i\left(A, P_{r}, P\right)=1, \quad \forall 0<r \leq r_{0},
$$

where $P_{r}=\{x \in P:\|u\|<r\}$.

Lemma 2.6 ([33]) Let $P$ be a cone in a Banach space $E, A: P \rightarrow P$ be completely continuous. Suppose that $A$ is differentiable at $\infty$ along $P$ and 1 is not an eigenvalue of $A_{+}^{\prime}(\infty)$ corresponding to a positive eigenvector. Moreover, if $A_{+}^{\prime}(\infty)$ has no positive eigenvectors corresponding to an eigenvalue greater than one, then there exists $R_{0}>0$ such that

$$
i\left(A, \Omega_{R}, P\right)=1, \quad \forall R_{0} \leq R,
$$

where $\Omega_{R}=\{x \in P:\|u\|<R\}$.

Lemma 2.7 ([34]) Let $k(x, y)$ be nonnegative on $[a, b] \times[a, b]$, and let the operator $K$ : $C[a, b] \rightarrow C[a, b]$ be completely continuous, where $K$ is defined as $K_{\phi}=\int_{[a, b]} k(x, y) \phi(y) d y$. If the spectral radius $r(A) \neq 0$, then $K$ has a positive eigenfunction $\phi_{1}$ corresponding to its first eigenvalue $\lambda_{1}=(r(A))^{-1}$, i.e. there exists $\phi_{1}(t) \in C[a, b]$ with $\lambda_{1} K \phi_{1}=\phi_{1}, \phi_{1}(t) \geq 0$, $\phi_{1}(t) \not \equiv 0, t \in[a, b]$.

\section{Main results}

Let $E_{0}=C([\eta, 1], \mathbb{R}),\|u\|_{0}=\max _{t \in[\eta, 1]}|u(t)|$. Define an operator $T_{0}$ on $E_{0}$ as

$$
T_{0} u(t)=\int_{\eta}^{1} G(t, s) u(s) d t, \quad t \in[\eta, 1]
$$

for $u \in E_{0}$, where $G(t, s)$ is the Green function (2.2), whose domain is restricted on $[\eta, 1] \times$ $[0,1]$.

Let $P_{0}=\left\{u \in E_{0}: u(t) \geq \omega_{0}\|u\|_{0}, t \in[\eta, 1]\right\}$, where $\omega_{0}$ is given in Lemma 2.3. Obviously, $P_{0}$ is a cone in $E_{0}$. We have the following lemma.

Lemma 3.1 $T_{0}: E_{0} \rightarrow E_{0}$ is completely continuous. Moreover, the spectral radius $r\left(T_{0}\right)>0$.

Proof Since the proof of the complete continuity of $T_{0}$ is similar to that in Lemma 2.4, we omit it. Here, we only show that $r\left(T_{0}\right)>0$.

Let $\phi(t)=\int_{\eta}^{1} G(t, s) d s, t \in[\eta, 1]$. Because

$$
\phi(t)=\frac{k}{k-1} t^{\alpha-1}(1-\eta)-\frac{1}{\alpha}(t-\eta)^{\alpha}, \quad t \in[\eta, 1]
$$


and

$$
\begin{aligned}
\phi^{\prime}(t) & =\frac{k}{k-1}(\alpha-1) t^{\alpha-2}(1-\eta)-(t-\eta)^{\alpha-1} \\
& >t^{\alpha-1}(1-\eta)-(t-\eta)^{\alpha-1}>0, \quad t \in[\eta, 1],
\end{aligned}
$$

we have

$$
\min _{t \in[\eta, 1]} \phi(t)=\phi(\eta)=\frac{k}{k-1} \eta^{\alpha-1}(1-\eta) \triangleq \gamma_{0}>0 .
$$

For any $u \in P_{0} \backslash\{\theta\}$, from

$$
\begin{aligned}
\left(T_{0} u\right)(t) & =\int_{\eta}^{1} G(t, s) u(s) d s \geq \omega_{0}\|u\|_{0} \int_{\eta}^{1} G(t, s) d s \\
& \geq \omega_{0} \gamma_{0}\|u\|_{0} \geq \omega_{0} \gamma_{0} u(t), \quad t \in[\eta, 1],
\end{aligned}
$$

we have

$$
\left(T_{0}^{2} u\right)(t)=T_{0}\left(T_{0} u\right) \geq \omega_{0} \gamma_{0} T_{0} u \geq\left(\omega_{0} \gamma_{0}\right)^{2} u(t), \quad t \in[\eta, 1]
$$

and so

$$
\left\|T_{0}^{n} u\right\|_{0} \geq\left(T_{0}^{n} u\right)(t) \geq\left(\omega_{0} \gamma_{0}\right)^{n} u(t), \quad t \in[\eta, 1]
$$

Hence, $\left\|T_{0}^{n} u\right\|_{0} \geq\left(\omega_{0} \gamma_{0}\right)^{n}\|u\|_{0}$, and so $\frac{\left\|T_{0}^{n} u\right\|_{0}}{\|u\|_{0}} \geq\left(\omega_{0} \gamma_{0}\right)^{n}$, which implies that $\left\|T_{0}^{n}\right\| \geq$ $\left(\omega_{0} \gamma_{0}\right)^{n}$. So, we obtain

$$
r\left(T_{0}\right)=\varlimsup_{n \rightarrow \infty}\left\|T^{n}\right\|^{\frac{1}{n}} \geq \omega_{0} \gamma_{0}>0 .
$$

The proof is complete.

Let us list the following assumptions, which will be used later.

$\left(\mathrm{H}_{1}\right) f \in C\left([0,1] \times \mathbb{R}_{+}, \mathbb{R}_{+}\right)$.

$\left(\mathrm{H}_{2}\right) f(t, 0)=0, f_{x}(t, 0) \geq 0, t \in[0,1], f_{x}(t, 0) \not \equiv 0, t \in[\eta, 1]$, where the partial derivative $f_{x}(t, 0)=\left.f_{x}(t, x)\right|_{(t, 0)}$. Moreover, $\exists b>0$ such that $f_{x}(t, x)$ is continuous on $[0,1] \times[0, b]$.

$\left(\mathrm{H}_{3}\right) \int_{0}^{1}\left(1-(1-s)^{\alpha-1}\right) f_{x}(s, 0) d s<\frac{\Gamma(\alpha)}{1+k_{0}}$, where $k_{0}=\frac{1}{(k-1)\left(1-(1-\eta)^{\alpha-1}\right)}, f_{x}(s, 0)=\left.f_{x}(s, x)\right|_{(s, 0)}$.

$\left(\mathrm{H}_{4}\right)$ There exists $\phi \in C\left([0,1], \mathbb{R}_{+}\right)$such that $\lim _{x \rightarrow+\infty} \frac{f(t, x)}{x}=\phi(t)$ holds uniformly on $[0,1]$ with respect to $t$. Moreover, $\phi(t) \not \equiv 0, t \in[\eta, 1]$.

$\left(\mathrm{H}_{5}\right) \int_{0}^{1}\left(1-(1-s)^{\alpha-1}\right) \phi(s) d s<\frac{\Gamma(\alpha)}{1+k_{0}}$, where $k_{0}=\frac{1}{(k-1)\left(1-(1-\eta)^{\alpha-1}\right)}$.

Define an operator $A$ on $P$ as

$$
(A u)(t)=\int_{0}^{1} G(t, s) f(s, u(s)) d s, \quad t \in[0,1] \text {, for } u \in P
$$

Obviously, the following lemma is true in view of Lemma 2.4. 
Lemma 3.2 Let $\left(\mathrm{H}_{1}\right)$ hold. Then $A: P \rightarrow P$ is completely continuous.

We need the following two lemmas, which will play an important role to obtain the existence results.

Lemma 3.3 Let $\left(\mathrm{H}_{1}\right)-\left(\mathrm{H}_{2}\right)$ hold. Then the operator $A$ is differentiable at $\theta$ along $P$, and $A \theta=\theta, A_{+}^{\prime}(\theta) h=B h, h \in P$, where

$$
B h=\int_{0}^{1} G(t, s) f_{x}(s, 0) h(s) d s, \quad h \in P .
$$

Proof For any $(s, x) \in[0,1] \times[0, b]$, by the mean value theorem, there exists $\xi \in(0, x)$ such that

$$
f(s, x)=f(s, x)-f(s, 0)=f_{x}(s, \xi) x,
$$

that is,

$$
f(s, x)-f_{x}(s, \xi) x=0
$$

Again, due to the fact that $f_{x}(s, x)$ is uniformly continuous on $[0,1] \times[0, b]$, for arbitrary $\varepsilon>0$, there exists $\delta \in(0, b)$ such that

$$
\left|f_{x}(s, x)-f_{x}(s, 0)\right|<\frac{\alpha \Gamma(\alpha)}{(\alpha-1)\left(1+k_{0}\right)} \varepsilon,
$$

when $0<x<\delta$, for all $s \in[0,1]$.

So, from (3.1)-(3.2), it follows that

$$
\begin{aligned}
\left|f(s, x)-f_{x}(s, 0) x\right| & \leq\left|f(s, x)-f_{x}(s, \xi) x\right|+\left|f_{x}(s, \xi)-f_{x}(s, 0)\right| x \\
& <\frac{\alpha \Gamma(\alpha)}{(\alpha-1)\left(1+k_{0}\right)} \varepsilon x,
\end{aligned}
$$

when $0<x<\delta$, for all $s \in[0,1]$.

Consequently, for any $h \in P$ with $\|h\|<\delta$, from (3.3) and (2.9), it follows that

$$
\begin{aligned}
|(A h)(t)-(B h)(t)| & \leq \int_{0}^{1} G(t, s)\left|f(s, h(s))-f_{x}(s, 0) h(s)\right| d s \\
& \leq \frac{\alpha \Gamma(\alpha)}{(\alpha-1)\left(1+k_{0}\right)} \varepsilon\|h\| \int_{0}^{1} G(t, s) d s \\
& \leq \frac{\alpha}{\alpha-1} \varepsilon\|h\| \int_{0}^{1}\left(1-(1-s)^{\alpha-1}\right) d s=\varepsilon\|h\| .
\end{aligned}
$$

So, $\|A h-B h\| \leq \varepsilon\|h\|$, that is, $A_{+}^{\prime}(\theta) h=B h, h \in P$. The proof is complete.

Lemma 3.4 Let $\left(\mathrm{H}_{1}\right)-\left(\mathrm{H}_{3}\right)$ hold. Then $A_{+}^{\prime}(\theta)$ has no positive eigenvectors corresponding to an eigenvalue greater than or equal to one. 
Proof If not, then there exist a $\lambda_{0} \geq 1$ and $h_{0} \in P \backslash\{\theta\}$ with $\lambda_{0} h_{0}=A_{+}^{\prime}(\theta) h_{0}$, and so

$$
\begin{aligned}
h_{0}(t) & \leq \lambda_{0} h_{0}(t)=\left(A_{+}^{\prime}(\theta) h\right)(t)=\int_{0}^{1} G(t, s) f_{x}(s, 0) h_{0}(s) d s \\
& \leq \frac{1+k_{0}}{\Gamma(\alpha)} \int_{0}^{1}\left(1-(1-s)^{\alpha-1}\right) f_{x}(s, 0) h_{0}(s) d s .
\end{aligned}
$$

Thus,

$$
\begin{aligned}
\left(1-(1-t)^{\alpha-1}\right) f_{x}(t, 0) h_{0}(t) \leq & \frac{1+k_{0}}{\Gamma(\alpha)}\left(1-(1-t)^{\alpha-1}\right) f_{x}(t, 0) \\
& \times \int_{0}^{1}\left(1-(1-s)^{\alpha-1}\right) f_{x}(s, 0) h_{0}(s) d s,
\end{aligned}
$$

and so

$$
\begin{aligned}
\int_{0}^{1}\left(1-(1-t)^{\alpha-1}\right) f_{x}(t, 0) h_{0}(t) d t \leq & \frac{1+k_{0}}{\Gamma(\alpha)} \int_{0}^{1}\left(1-(1-t)^{\alpha-1}\right) f_{x}(t, 0) d t \\
& \times \int_{0}^{1}\left(1-(1-s)^{\alpha-1}\right) f_{x}(s, 0) h_{0}(s) d s .
\end{aligned}
$$

Because $h_{0} \in P \backslash\{\theta\}, f_{x}(t, 0) \geq 0, t \in[0,1], f_{x}(t, 0) \not \equiv 0, t \in[\eta, 1]$ and $f_{x}(t, 0)$ is continuous on $[0,1]$, the following inequality:

$$
\int_{0}^{1}\left(1-(1-t)^{\alpha-1}\right) f_{x}(t, 0) h_{0}(t) d t>0
$$

holds. Immediately, from (3.4) it follows that $\frac{1+k_{0}}{\Gamma(\alpha)} \int_{0}^{1}\left(1-(1-t)^{\alpha-1}\right) f_{x}(t, 0) d t \geq 1$, which contradicts $\left(\mathrm{H}_{3}\right)$. So, the conclusion of Lemma 3.4 is true. The proof is complete.

Lemma 3.5 Let $\left(\mathrm{H}_{1}\right),\left(\mathrm{H}_{4}\right)$, and $\left(\mathrm{H}_{5}\right)$ hold. Then $A$ is differentiable at $\infty$ along $P$ and $A_{+}^{\prime}(\infty) h=G h, h \in P$, where

$$
G h=\int_{0}^{1} G(t, s) \phi(s) h(s) d s, \quad h \in P .
$$

Proof From $\left(\mathrm{H}_{4}\right)$, it follows that, for arbitrary $\varepsilon>0$, there exists $R>0$ such that

$$
\left|\frac{f(t, x)}{x}-\phi(t)\right|<\varepsilon, \quad t \in[0,1],
$$

when $x>R$, and so

$$
|f(t, x)-\phi(t) x|<\varepsilon x, \quad t \in[0,1],
$$

when $x>R$.

Let $M=\sup _{(t, x) \in[0,1] \times[0, R]}|f(t, x)-\phi(t) x|$. Then

$$
|f(t, x)-\phi(t) x|<M+\varepsilon x, \quad t \in[0,1]
$$

holds for any $x \in \mathbb{R}_{+}$. 
Now, for any $h \in P$, by the above inequality, we have

$$
\begin{aligned}
|(A h)(t)-(G h)(t)| & \leq \int_{0}^{1} G(t, s)|f(s, h(s))-\phi(s) h(s)| d s \\
& \leq(M+\varepsilon\|h\|) \int_{0}^{1} G(t, s) d s \\
& \leq(M+\varepsilon\|h\|) L, \quad t \in[0,1],
\end{aligned}
$$

where $L=\max _{t \in[0,1]} \int_{0}^{1} G(t, s) d s$. Thus $\frac{\|A h-G h\|}{\|h\|} \leq \frac{M L}{\|h\|}+L \varepsilon$. So, $\frac{\|A h-G h\|}{\|h\|}<2 L \varepsilon$ when $\|h\|>\frac{M}{\varepsilon}$, that is, $A_{+}^{\prime}(\infty) h=G h$. The proof is complete.

Lemma 3.6 Let $\left(\mathrm{H}_{1}\right),\left(\mathrm{H}_{4}\right)$, and $\left(\mathrm{H}_{5}\right)$ hold. Then $A_{+}^{\prime}(\infty)$ has no positive eigenvectors corresponding to an eigenvalue greater than or equal to one.

Proof The proof is similar to the proof of Lemma 3.4. In fact, if not, then there exist a $\lambda_{0} \geq 1$ and $h_{0} \in P \backslash\{\theta\}$ such that $\lambda_{0} h_{0}=A_{+}^{\prime}(\infty) h_{0}$, and so

$$
\begin{aligned}
h_{0}(t) & \leq \lambda_{0} h_{0}(t)=\left(A_{+}^{\prime}(\infty) h_{0}\right)(t)=\int_{0}^{1} G(t, s) \phi(s) h_{0}(s) d s \\
& \leq \frac{1+k_{0}}{\Gamma(\alpha)} \int_{0}^{1}\left(1-(1-s)^{\alpha-1}\right) \phi(s) h_{0}(s) d s .
\end{aligned}
$$

Thus,

$$
\left(1-(1-t)^{\alpha-1}\right) \phi(t) h_{0}(t) \leq \frac{1+k_{0}}{\Gamma(\alpha)}\left(1-(1-t)^{\alpha-1}\right) \phi(t) \int_{0}^{1}\left(1-(1-s)^{\alpha-1}\right) \phi(s) h_{0}(s) d s
$$

and so

$$
\begin{aligned}
\int_{0}^{1}\left(1-(1-t)^{\alpha-1}\right) \phi(t) h_{0}(t) d t \leq & \frac{1+k_{0}}{\Gamma(\alpha)} \int_{0}^{1}\left(1-(1-t)^{\alpha-1}\right) \phi(t) d t \\
& \times \int_{0}^{1}\left(1-(1-s)^{\alpha-1}\right) \phi(s) h_{0}(s) d s
\end{aligned}
$$

Because $h_{0} \in P \backslash\{\theta\}$ and $\phi(t) \not \equiv 0, t \in[\eta, 1], \phi \in C\left([0,1], \mathbb{R}_{+}\right)$, the relation

$$
\int_{0}^{1}\left(1-(1-t)^{\alpha-1}\right) \phi(t) h_{0}(t) d t>0
$$

holds, and therefore $\frac{1+k_{0}}{\Gamma(\alpha)} \int_{0}^{1}\left(1-(1-t)^{\alpha-1}\right) \phi(t) d t \geq 1$, which contradicts $\left(\mathrm{H}_{5}\right)$. The proof is complete.

Let $f_{\infty}=\lim _{x \rightarrow \infty} \inf \min _{t \in[\eta, 1]} \frac{f(t, x)}{x}$. We are in a position to state our main result in the present paper.

Theorem 3.1 Let $\left(\mathrm{H}_{1}\right)-\left(\mathrm{H}_{3}\right)$ hold. If $f_{\infty}>r^{-1}\left(T_{0}\right)$, then BVP (1.1)-(1.2) has a positive solution. 
Proof In view of Lemmata 3.2-3.4 and by applying Lemma 2.5, we conclude that there exist a $r_{0}>0$ such that

$$
i\left(A, \Omega_{r_{0}}, P\right)=1
$$

where $\Omega_{r}=P \cap B_{r}, B_{r}=\{x \in E:\|u\|<r\}$ and $P$ is defined as before.

By Lemma 2.7 and Lemma 3.1, we know that there exists a $\phi_{1} \in C[\eta, 1]$ with $\phi_{1}(t) \geq 0$, $\phi_{1}(t) \not \equiv 0, t \in[\eta, 1]$ satisfying

$$
r^{-1}\left(T_{0}\right) \int_{\eta}^{1} G(t, s) \phi_{1}(s) d s=\phi_{1}(t), \quad t \in[\eta, 1] .
$$

So, from Lemma 2.3, it follows that

$$
\phi_{1}(t) \geq \omega_{0} r^{-1}\left(T_{0}\right) \int_{\eta}^{1} G(\tau, s) \phi_{1}(s) d s=\omega_{0} \phi_{1}(\tau), \quad t, \tau \in[\eta, 1]
$$

Thus, $\phi_{1}(t) \geq \omega_{0}\left\|\phi_{1}\right\|_{0}, t \in[\eta, 1]$, that is, $\phi_{1} \in P_{0}$.

Let

$$
\phi_{0}(t)= \begin{cases}\phi_{1}(\eta), & t \in[0, \eta] \\ \phi_{1}(t), & t \in[\eta, 1]\end{cases}
$$

It is easy to see that $\phi_{1} \in P$.

On the other hand, by $f_{\infty}>r^{-1}\left(T_{0}\right)$, there exists a $R>0$ such that

$$
f(t, x)>r^{-1}\left(T_{0}\right) x, \quad t \in[\eta, 1]
$$

when $x \geq R$. Take $R_{0} \geq \max \left\{\omega_{0}^{-1} R, r_{0}\right\}$. Set $\Omega_{R_{0}}=\left\{u \in P:\|u\|<R_{0}\right\}$. Then for any $u \in \partial \Omega_{R_{0}}$, the inequality $u(t) \geq \omega_{0}\|u\|=\omega_{0} R_{0} \geq R, t \in[\eta, 1]$ implies

$$
f(t, u(t))>r^{-1}\left(T_{0}\right) u(t), \quad t \in[\eta, 1] .
$$

We show that the following relation holds:

$$
u(t)-(A u)(t) \neq \lambda \phi_{0}(t), \quad \forall \lambda \geq 0, \forall u \in \partial \Omega_{R_{0}}, t \in[0,1]
$$

In fact, if not, then there exist a $u_{0} \in \partial \Omega_{R_{0}}$ and a $\lambda_{0} \geq 0$ such that

$$
u_{0}(t)=\left(A u_{0}\right)(t)+\lambda_{0} \phi_{0}(t), \quad t \in[0,1] .
$$

Obviously, we can assume that $\lambda_{0}>0$. From (3.8), it follows that

$$
u_{0}(t) \geq \lambda_{0} \phi_{0}(t), \quad t \in[0,1]
$$

because $A P \subset P$. 
Let $\lambda^{*}=\sup \left\{\lambda \mid u_{0}(t) \geq \lambda \phi_{0}(t), t \in[\eta, 1]\right\}$. Then $0<\lambda^{*}<+\infty$ and

$$
u_{0}(t) \geq \lambda^{*} \phi_{0}(t), \quad t \in[\eta, 1]
$$

Again, from (3.8), (3.6), and (3.9), for $t \in[\eta, 1]$, we have

$$
\begin{aligned}
u_{0}(t) & =\left(A u_{0}\right)(t)+\lambda_{0} \phi_{0}(t) \\
& =\int_{0}^{1} G(t, s) f\left(s, u_{0}(s)\right) d s+\lambda_{0} \phi_{0}(t) \\
& \geq \int_{\eta}^{1} G(t, s) f\left(s, u_{0}(s)\right) d s+\lambda_{0} \phi_{0}(t) \\
& >r^{-1}\left(T_{0}\right) \int_{\eta}^{1} G(t, s) u_{0}(s) d s+\lambda_{0} \phi_{0}(t) \\
& \geq r^{-1}\left(T_{0}\right) \lambda^{*} \int_{\eta}^{1} G(t, s) \phi_{0}(s) d s+\lambda_{0} \phi_{0}(t) \\
& =r^{-1}\left(T_{0}\right) \lambda^{*}\left(T_{0} \phi_{0}\right)(t)+\lambda_{0} \phi_{0}(t) \\
& =\left(\lambda^{*}+\lambda_{0}\right) \phi_{0}(t), \quad t \in[\eta, 1],
\end{aligned}
$$

which contradicts the definition of $\lambda^{*}$. Hence, the relation (3.7) holds. So, in terms of the fixed point index theorem on a cone, we have

$$
i\left(A, \Omega_{R_{0}}, P\right)=0
$$

Thus, (3.5) and (3.10) imply that

$$
i\left(A, \Omega_{R_{0}} \backslash \bar{\Omega}_{r_{0}}, P\right)=-1 .
$$

So, $A$ has a fixed point $\bar{u} \in \Omega_{R_{0}} \backslash \bar{\Omega}_{r_{0}}$, that is, $\bar{u}$ is a positive solution of BVP (1.1)-(1.2). The proof is complete.

Let $\mu_{0}=\frac{\Gamma(\alpha)}{\omega_{0}} \frac{k-1}{k} \eta^{1-\alpha}(1-\eta)^{-1}$, where $\omega_{0}$ is given as in Lemma 2.3. We state another result in this paper.

Theorem 3.2 Let $\left(\mathrm{H}_{1}\right),\left(\mathrm{H}_{4}\right)$, and $\left(\mathrm{H}_{5}\right)$ hold. Assume that there exists $r_{0}$ such that

$$
f(t, x) \geq \mu_{0} x, \quad x \in\left[0, r_{0}\right], t \in[0,1] .
$$

Then BVP (1.1)-(1.2) has a positive solution.

Proof We show that

$$
\|A u\| \geq\|u\|, \quad \forall u \in \Omega_{r_{0}}
$$

where $\Omega_{r_{0}}=\left\{u \in P:\|u\|<r_{0}\right\}$. 
In fact, for any $u \in \partial \Omega_{r_{0}}$, from (3.11) we have

$$
f(t, u(t)) \geq \mu_{0} u(t), \quad t \in[0,1]
$$

owing to

$$
0 \leq u(t) \leq r_{0}, \quad t \in[0,1]
$$

Thus, by Lemma 2.3,

$$
\begin{aligned}
\|A u\| & \geq(A u)(t)=\int_{0}^{1} G(t, s) f(s, u(s)) d s \\
& \geq \mu_{0} \int_{0}^{1} G(t, s) u(s) d s \\
& \geq \mu_{0} \omega_{0}\|u\| \int_{0}^{1} G(\tau, s) d s \\
& \geq \mu_{0} \omega_{0}\|u\| \int_{\eta}^{1} G(\tau, s) d s, \quad \tau \in[0,1], t \in[\eta, 1] .
\end{aligned}
$$

So,

$$
\|A u\| \geq \mu_{0} \omega_{0}\|u\| \int_{\eta}^{1} G(\eta, s) d s=\|u\|,
$$

because

$$
\omega_{0} \int_{\eta}^{1} G(\eta, s) d s=\frac{\omega_{0}}{\Gamma(\alpha)} \frac{k}{k-1} \eta^{\alpha-1}(1-\eta)=\mu_{0}^{-1}
$$

Therefore, the relation (3.12) holds. Consequently, applying the fixed point index theorem, we get

$$
i\left(A, \Omega_{r_{0}}, P\right)=0 \text {. }
$$

On the other hand, by Lemma 3.2, Lemma 3.5, Lemma 3.6, and Lemma 2.6, we know that there exists $R_{0}>r_{0}$ such that

$$
i\left(A, \Omega_{R_{0}}, P\right)=1 \text {. }
$$

So, by (3.13) and (3.14), we have

$$
i\left(A, \Omega_{R_{0}} \backslash \bar{\Omega}_{r_{0}}, P\right)=-1 .
$$

Therefore, $A$ has a fixed point $\bar{u} \in \Omega_{R_{0}} \backslash \bar{\Omega}_{r_{0}}$, that is, $\bar{u}$ is a positive solution of BVP (1.1)(1.2). The proof is complete. 
Example 3.1 Consider the following boundary value problem:

$$
\left\{\begin{array}{l}
D_{0^{+}}^{\frac{5}{2}} u(t)=\ln \left(1+t^{2}\right) \sin ^{2} u(t)+t^{2} \frac{u(t) e^{u(t)}}{1+u^{2}(t)}, \quad t \in(0,1), \\
u(0)=u^{\prime}(0)=0, \quad D_{0^{+}}^{\frac{3}{2}} u\left(\frac{1}{2}\right)=2 D_{0^{+}}^{\frac{3}{2}} u(1) .
\end{array}\right.
$$

To obtain the existence result, we will apply Theorem 3.1 with $\alpha=\frac{5}{2}, n=3, \eta=\frac{1}{2}, k=2$ and function $f(t, x)=\ln \left(1+t^{2}\right) \sin ^{2} x+\frac{t^{2} x e^{x}}{1+x^{2}}, t \in[0,1], x \in[0,+\infty)$. Clearly, the function $f(t, x)$ satisfies $f \in C\left([0,1] \times \mathbb{R}_{+}, \mathbb{R}_{+}\right), f(t, 0)=0, f_{x}(t, x)=\ln \left(1+t^{2}\right) \sin 2 x+t^{2} \frac{e^{x}\left(1+x-x^{2}+x^{3}\right)}{\left(1+x^{2}\right)^{2}}$ and $f_{x}(t, 0)=t^{2}>0, t \in\left[\frac{1}{2}, 1\right]$. Further, $\int_{0}^{1}\left(1-(1-s)^{\frac{3}{2}}\right) f_{x}(s, 0) d s=\frac{89}{315}<\frac{3(15-2 \sqrt{2})}{124} \sqrt{\pi}=\frac{\Gamma(\alpha)}{1+k_{0}}$ and $f_{\infty}=+\infty$. So, all the assumptions of Theorem 3.1 are satisfied and therefore BVP (3.15) has at least one positive solution.

Example 3.2 Consider the following boundary value problem:

$$
\left\{\begin{array}{l}
D_{0^{+}}^{\frac{5}{2}} u(t)=\frac{15}{42} e^{t}(1+u(t))+\ln (2+t)|\cos u(t)|, \quad t \in(0,1), \\
u(0)=u^{\prime}(0)=0, \quad D_{0^{+}}^{\frac{3}{2}} u\left(\frac{1}{2}\right)=2 D_{0^{+}}^{\frac{3}{2}} u(1) .
\end{array}\right.
$$

To obtain the existence result, we will apply Theorem 3.2 with $\alpha=\frac{5}{2}, n=3, \eta=\frac{1}{2}, k=$ 2 and function $f(t, x)=\frac{15}{42} e^{t}(1+x)+\ln (2+t)|\cos x|, t \in[0,1], x \in[0,+\infty)$. Clearly, the function $f(t, x)$ satisfies $f \in C\left([0,1] \times \mathbb{R}_{+}, \mathbb{R}_{+}\right), \lim _{x \rightarrow+\infty} \frac{f(t, x)}{x} \doteq \phi(t)=\frac{15}{42} e^{t}$ holds uniformly on $[0,1]$ with respect to $t$. Further, $\int_{0}^{1}\left(1-(1-s)^{\frac{3}{2}}\right) \phi(s) d s<\frac{9}{14}<\frac{3(15-2 \sqrt{2})}{124} \sqrt{\pi}=\frac{\Gamma(\alpha)}{1+k_{0}}$. On the other hand, it is easy to see that exists a $r_{0} \in\left(0, \frac{\pi}{2}\right)$ such that $f(t, x) \geq \mu_{0} x, x \in\left[0, r_{0}\right], t \in$ $[0,1]$ noting that $f(t, x)>(\ln 2) \cos x, x \in\left[0, \frac{\pi}{2}\right], t \in[0,1]$ and $\left.\left((\ln 2) \cos x-\mu_{0} x\right)\right|_{x=0}=\ln 2$, $\left((\ln 2) \cos x-\mu_{0} x\right)^{\prime}<0, x \in\left[0, \frac{\pi}{2}\right], t \in[0,1]$. So, all the assumptions of Theorem 3.2 are satisfied and therefore BVP (3.16) has at least one positive solution.

\section{Competing interests}

The authors declares that they have no competing interests.

\section{Authors' contributions}

The authors contributed equally in this article. All authors read and approved the final manuscript.

\section{Acknowledgements}

The authors sincerely thank the anonymous referees for their valuable suggestions and comments, which have greatly helped improve this article. This work is supported by the Natural Science Foundation of Hubei Provincial Education Department (Q20132505).

\section{Received: 9 January 2014 Accepted: 4 March 2014 Published: 18 Mar 2014}

\section{References}

1. Samko, SG, Kilbas, AA, Marichevand, OI: Fractional Integrals and Derivatives Theory and Application. Gordon \& Breach, Yverdon (1993)

2. Podlubny, I: Fractional Differential Equations. Academic Press, San Diego (1999)

3. Kilbas, AA, Srivastava, HM, Trujillo, JJ: Theory and Applications of Fractional Differential Equations. North-Holland Mathematics Studies, vol. 204. Elsevier, Amsterdam (2006)

4. Lakshmikantham, V, Leela, S, Vasundhara, J: Theory of Fractional Dynamic Systems. Cambridge Academic Publishers, Cambridge (2009)

5. Diethelm, K: The Analysis of Fractional Differential Equations. Springer, New York (2010)

6. Xu, J, Wei, Z, Dong, W: Uniqueness of positive solutions for a class of fractional boundary value problems. Appl. Math Lett. 25, 590-593 (2012)

7. Vong, SW: Positive solutions of singular fractional equations with integral boundary conditions. Math. Comput. Model. 57, 1053-1059 (2013)

8. Yang, L, Zhang, W, Liu, X: A sufficient condition for the existence of a positive solutions for a nonlinear fractional differential equation with the Riemann-Liouville derivative. Appl. Math. Lett. 25, 1986-1992 (2012) 
9. Zhang, $X$, Han, Y: Existence and uniqueness of positive solutions for higher order nonlocal fractional differential equations. Appl. Math. Lett. 25, 555-560 (2012)

10. $W u, J, Z$ hang, $X, L i u, L, W u, Y$ : Positive solutions of higher-order nonlinear fractional differential equations with changing sign measure. Adv. Differ. Equ. 2012, Article ID 71 (2012)

11. Agarwal, RP, Ntouyas, SK, Ahmad, B, Alhothuali, MS: Existence of solutions for integro-differential equations of fractional order with nonlocal three-point fractional boundary conditions. Adv. Differ. Equ. 2013, Article ID 128 (2013)

12. Liu, Y, Ahmad, B, Agarwal, RP: Existence of solutions for a coupled system of nonlinear fractional differential equations with fractional boundary conditions on the half-line. Adv. Differ. Equ. 2013, Article ID 46 (2013)

13. Zhang, $X, L i u, L, W u, Y, L u, Y: T h e$ iterative solutions of nonlinear fractional differential equations. Appl. Math. Comput. 219, 4680-4691 (2013)

14. Zhai, C, Yan, W, Yang, C: A sum operator method for existence and uniqueness of positive solutions to Riemann-Liouville fractional differential equation boundary value problems. Commun. Nonlinear Sci. Numer. Simul. 18, 858-866 (2013)

15. Chai, G: Anti-periodic boundary value problems of fractional differential equations with the Riemann-Liouville fractional derivative. Adv. Differ. Equ. 2013, Article ID 306 (2013)

16. Ahmad, B, Alsaedi, A, Assolami, A, Agarwal, RP: A study of Riemann-Liouville fractional nonlocal integral boundary value problems. Bound. Value Probl. 2013, Article ID 274 (2013)

17. Chai, G: Existence results of positive solutions for boundary value problems of fractional differential equations. Bound. Value Probl. 2013, Article ID 109 (2013)

18. Ahmad, B, Ntouyas, SK, Alsaedi, A: New results for boundary value problems of Hadamard-type fractional differential inclusions and integral boundary conditions. Bound. Value Probl. 2013, Article ID 275 (2013)

19. Chalishajar, DN, Karthikeyan, K: Existence and uniqueness results for boundary value problems of higher order fractional integro-differential equations involving Gronwall's inequality in Banach spaces. Acta Math. Sci. 33(3), 758-772 (2013)

20. Chai, G: Positive solutions for boundary value problem of fractional differential equation with $p$-Laplacian operator. Bound. Value Probl. 2012, Article ID 18 (2012)

21. Liu, Y: Existence of solutions for impulsive differential models on half lines involving Caputo fractional derivatives. Commun. Nonlinear Sci. Numer. Simul. 18, 2604-2625 (2013)

22. Rehman, MU, Eloe, PW: Existence and uniqueness of solutions for impulsive fractional differential equations. Appl. Math. Comput. 224, 422-431 (2013)

23. Chai, G: Existence results for anti-periodic boundary value problems of fractional differential equations. Adv. Differ. Equ. 2013, Article ID 53 (2013)

24. Jankowski, T: Boundary problems for fractional differential equations. Appl. Math. Lett. 28, 14-19 (2014)

25. Baleanu, D, Agarwal, RP, Mohammadi, H, Rezapour, S: Some existence results for a nonlinear fractional differential equation on partially ordered Banach spaces. Bound. Value Probl. 2013, Article ID 112 (2013)

26. Liu, Y: Positive solutions using bifurcation techniques for boundary value problems of fractional differential equations. Abstr. Appl. Anal. 2013, Article ID 162418 (2013)

27. Darzi, R, Mohammadzadeh, B, Neamaty, A, Baleanu, D: Lower and upper solutions method for positive solutions of fractional boundary value problems. Abstr. Appl. Anal. 2013, Article ID 847184 (2013)

28. Goodrich, CS: Existence of a positive solution to a class of fractional differential equations. Appl. Math. Lett. 23, 1050-1055 (2010)

29. Goodrich, CS: Existence of a positive solution to systems of differential equations of fractional order. Comput. Math. Appl. 62, 1251-1268 (2011)

30. Wang, J, Xiang, H, Zhao, Y: Monotone and concave positive solutions to a boundary value problem for higher-order fractional differential equation. Abstr. Appl. Anal. 2011, Article ID 430457 (2011)

31. El-Shahed, M, Shammakh, WM: Existence of positive solutions for $m$-point boundary value problem for nonlinear fractional differential equation. Abstr. Appl. Anal. 2011, Article ID 986575 (2011)

32. Bai, Z, Lü, H: Positive solutions for boundary value problem of nonlinear fractional differential equation. J. Math. Anal. Appl. 311, 495-505 (2005)

33. Guo, D, Lakshmikantham, V: Nonlinear Problems in Abstract Cones. Academic Press, New York (1988)

34. Guo, D, Sun, J: Nonlinear Integral Equations. Shandong Science and Technology Press, Jinan (1987) (in Chinese)

10.1186/1687-1847-2014-90

Cite this article as: Chai and Hu: Existence of positive solutions for a fractional high-order three-point boundary

value problem. Advances in Difference Equations 2014, 2014:90 